\title{
LES PHOSPHATES DE CALCIUM DU LAIT
}

\author{
par \\ Mue J. BRIGANDO \\ et MM. CHAMP et CLOSSON \\ Docteur de l'Université de Lyon \\ Chimistes à la Société des Caves
}

Dans son livre : "Le lait au point de vue colloïdal ", Ch. Porcher distingue nettement le rôle des sels solubles et des sels insolubles de calcium dans le phénomène de l'emprésurage.

Si les premiers sont indispensables à la coagulation par le lab, les sels insolubles de calcium ne doivent pas être négligés; ils interviennent comme constituant des caillés formés de caséinate de calcium et de phosphates bi et tricalcique.

Dans un lait, les proportions relatives de phosphates monocalcique, bicalcique et tricalcique sont variables et susceptibles de modifieations constantes, en rapport avec l'acidification du milieu.

Dans un travail réeent, publié à la Société de Chimie Industrielle (1), travail qui est une application des recherches du Professeur Ch. Porcher, et la transposition d'essais de laboratoire à la technique industrielle, il a été montré que la texture des eaillés, - si importante en fromagerie, est conditionnée par leur teneur en phosphates insolubles de calcium. En raison de l'importance au point de vue pratique de la teneur des laits en sels solubles et insolubles de calcium, il nous paraît intéressant d'étudier les facteurs qui modifient les phosphates de calcium du lait et d'examiner les différents cas devant lesquels peut se trouver l'industriel, et que nous groupons en : laits acides, laits " paresseux" et laits chauffés.

Les laits acides. - Quand un lait s'acidifie, l'acide lactique formé aux dépens du lactose déminéralise les sels du lait. Les phosphates tricalciques sont transformés en phosphates bi et monocalciques, en même temps qu'il se forme des lactates acides de calcium. Le caséinate de calcium est parallèlement décalcifié.

Selon l'acidité du lait, il est possible d'envisager tous les états intermédiaires entre un lait normal et un lait très acide où les phosphates sont presque entièrement à l'état de phosphates monocalciques et où la caséine, en partie déminéralisée, est en imminence de précipitation.

Les caillés résultant de l'emprésurage de tels laits présentent tous les degrés entre la texture normale et celle, bien particulière, du paracaséinate calcique, qui est "caoutchouteuse".

On sait l'importance que présente en technique fromagère un

(1) J. Brigando, Champ et Closson. Une application des travaux du Professeux Ch. Porcher à l'industrie fromagère : addition de phosphates de calcium colloïdaux au lait. $14^{e}$ Congrès de Chimie Industrielle, Section XV; 24 octobre 1934. 
bon égouttage du caillé. Un bon égouttage entraîne un développement normal de la flore microbienne, d'où résulte une bonne maturation des fromages.

Par suite d'une fabrication plus centralisée des fromages, laquelle nézess'te le transport des laits, et, d'autre part, du fait que le travail intensif des fromageries se présente surtout l'été, époque où les laits s'acidifient très facilement, le technicien, obligé, dans ces conditions, de traiter des laits acides, se trouve souvent en présence de caillés caoutchouteux, difficiles à égoutter.

Il est possible de modifier cette texture spéciale des caillés, par l'addition, aux laits acides, de phosphates de calcium riches en calcium, comme cela a été indiqué dans le travail publié à la Saciété de Chimie Industrielle, et ci-dessus mentionné.

A un lait titrant $28^{\circ}$ Dornic et de $p H 6,34$, il est ajouté un phosphate de caleium renfermant $0 \mathrm{gr}$. 39 de $\mathrm{P}^{2} \mathrm{O}^{5}$ et $0 \mathrm{gr}$. 667 de $\mathrm{CaO}$.

Le rapport $\frac{\mathrm{P}^{2} \mathrm{O}^{5}}{\mathrm{CaO}}$ est égal à 0,58 .

Le lait titre $22^{\circ} \mathrm{D}$. et son $p$ H est ramené à 6,67 .

Dans le phosphate tricalcique, le rapport $\frac{\mathrm{P}^{2} \mathrm{O}^{5}}{\mathrm{CaO}}$ étant de 0,845 , nous voyons que le phosphate ajouté est constitué par du phosphate tricalcique riche en chaux libre. On obtient un caillé non caoutchouteux, s'égouttant bien. Le fromage ainsi fabriqué avec du lait traité est d'une qualité bien supérieure à celle du fromage fabriqué à partir de lait non traité.

Les laits paresseux. - Il est des laits d'une acidité normale qui donnent à l'emprésurage des caillés mous, se travaillant mal. On les désigne en fromagerie sous le nom de laits " paresseux".

Ces laits, nous dit Ch. Porcher, proviennent le plus généralement, pour ne pas dire toujours, de mamelles malades. Selon l'intensité du processus qui frappe la mamelle, nous pouvons avoir des laits qui affectent des compositions chimiques des plus variables, se traduisant d'abord par une atteinte légère, caractérisée par une modification peu sensible des rapports quantitatifs des albumines et des globulines avec la caséine, puis par des troubles profonds, accompagnés d'une diminution considérable du taux de la caséine et d'une augmentation de celui de l'albumine et de la globuline.

En même temps que la caséine, les composants du complexe normal sont touchés parallèlement. S'il y a moins de caséinate, il y a également moins de phosphates de calcium, moins de citrates, et, d'une façon générale, moins de toutes les molécules spécifiquement élaborées par la cellule mammaire.

L'afflux leucocytaire qui intervient pour s'emparer des globules 
graisseux et digérer la caséine, ainsi qu'il en résulte des recherehes de E. Muffet (1), effectuées sous la direction de Ch. Porcher, donne naissance à des globulines. Les laits de mammites et les laits de rétention sont done caractérisés par une diminution des phosphates de calcium et du caséinate et une augmentation du taux des globulines et des albumines.

Du fait de la diminution du taux des phosphates de caleium, il était intéressant d'additionner ces laits de phosphates de calcium.

Le lait traité titre $20^{\circ} 5 \mathrm{D}$. et a un $p \mathrm{H}$ de 6,64; l'acidité est done normale, mais ce lait caille difficilement.

Il est ajouté un phosphate de calcium renfermant 0 gr. 33 de $\mathrm{P}^{2} \mathrm{O}^{5}$ et 0 gr. 20 de $\mathrm{CaO}$.

Le rapport $\frac{\mathrm{P}^{2} \mathrm{O}^{5}}{\mathrm{CaO}}$ est égal à 1,66 .

Après traitement, le lait titre $26^{\circ} \mathrm{D}$. et son $p$ H est de 6,62 .

Le rapport $\frac{\mathrm{P}^{2} \mathrm{O}^{5}}{\mathrm{CaO}}$ étant de 1,26 dans le phosphate bicalcique et de 2,52 dans le phosphate monocalcique, les phosphates ajoutés, de rapport $\frac{\mathrm{P}^{2} \mathrm{O}^{5}}{\mathrm{CaO}}$ égal à 1,66 , sont constitués principalement par un mélange de phosphates bi et monocalciques.

Nous devons, en effet, ajouter les phosphates insolubles qui manquent sous la forme de phosphate bicalcique, et, d'autre part, augmenter le taux des sels solubles de calcium sous forme de phosphate monocalcique, pour faciliter l'emprésurage, et contrebalancer l'action protectrice exercée par les globulines.

L'addition de phosphate monocalcique explique pourquoi, après le traitement, le lait a une acidité Dornic plus élevée. Le chiffre du $p H$ est légèrement plus faible, mais les variations sont petites, en raison même du milieu dans lequel on opère, qui est un milieutampon.

Le lait ainsi traité donne un fromage de pâte beaucoup plus ferme et plus fine que la pâte obtenue pour le fromage de lait non traité.

En raison même de la diminution du taux de la caséine, l'addition de caséinate de calcium en même temps que de phosphates de ealcium ne pourrait donner que des résultats satisfaisants.

Laits chauffés. - Les laits chauffés, même à la température de pasteurisation $\left(65-75^{\circ}\right)$, coagulent assez mal par la présure. Il en résulte que la pasteurisation des laits pour la fabrication des fromages, malgré l'avantage qu'elle peut présenter, savoir : détruire

(1) E. Murfer. Contribution à l'étude de la rétention lactée. Le sort de la caséine dans ce processus. Thèse Doct. Vét. Lyon, 1928. 
toute la flore indésirable des mauvais microbes, n'est pas encore entrée dans la pratique courante.

Au point de vue hygiénique, la pasteurisation des laits destinés à l'industrie fromagère, est chose éminemment souhaitable. Le bacille tuberculeux reste virulent plusieurs semaines, même dans les fromages fermentés. Le fragile bacille de Bang (agent de la fièvre méditerranéenne, ou fièvre ondulante), et, à plus forte raison, les autres germes pathogènes que peut contenir le lait, ne sont pas tués par la simple fermentation lactique caractérisant les fromages frais. Et nous nous rallions au vœu émis par M. le Doeteur MACHeB @UF, de l'Institut Pasteur, à savoir que, pour le moins, les laits destinés à la fabrication des fromages frais, devraient être pasteurisés. Mais pour souhaitable que soit, en général, la pasteurisation des laits destinés à l'industrie fromagère, il faut dire cependant que l'emploi en fromagerie des laits pasteurisés, nullement impossible sans doute, est loin d'être pratiquement réalisé. De nombreux essais ont déjà été tentés, tant en France qu'à l'Etranger. Les résultats ont été le plus souvent encourageants. L'intérêt économique considérable qui se superpose ici à l'intérêt hygiénique donne d'ailleurs à cette question un grand avenir.

Il résulte des travaux de Barillé (1) et de Ch. Porcher (2), que ce sont les phosphates de calcium du lait et l'albumine qui sont les plus touchés par le chauffage. La pasteurisation se traduit par une augmentation des phosphates insolubles de calcium et une diminution des phosphates solubles.

Dans ces conditions, l'addition aux laits pasteurisés d'un mélange de phosphates bi et monocalciques pourrait donner des résultats intéressants.

Ces laits devront naturellement être réensemencés avec des ferments lactiques de même qualité biochimique que ceux du lait originel, ainsi que l'ont préconisé Ch. Porcher et G. Thieulin (3).

\section{GONGLUSIONS}

Les sels de calcium, et en particulier les phosphates de calcium, doivent être envisagés spécialement à la faveur des altérations que subit le lait.

C'est ainsi que les laits acides, les laits paresseux et les laits chauffés sont caractérisés par une modification des proportions relatives des phosphates insolubles et des phosphates solubles de caleium.

(1) Barmutí. De l'existence des carbono-phosphates dans le lait. Leur précipitation par la pasteurisation. Société de Pharmacie, séance du 28 juillet 1909.

(2) Ch. Porcher. Action de la chaleur sur le complexe caséinate de chaux + phosphate de chaux. C. R. de l'A cadémie des Sciences, 1926, 186, 1247.

(3) † Ch. Porcher et G. ThIEvurn. Essais sur la fabrication du Port-Salut en partant de laits pasteurisés réensemencés de levain lactique. 
Ces altérations des laits peuvent être, en un certain sens, atténuées par l'addition correspondante de phosphates de calcium à l'état convenable.

Pour les laits acides, le phosphate à ajouter doit être riche en chaux, tandis qu'il doit être pauvre en chaux pourles laits paresseux.

Les fromages fabriqués à partir des laits traités s'affinent dans de très bonnes conditions. Leur maturation lente se produit sans l'apparition de goûts amers ou "putrides ". Ces fromages présentent à la coupe un très bon aspect et sont d'une qualité supérieure à celle des fromages préparés à partir des laits dont les altérations n'ont pas été corrigées.

\title{
REMARQUES SUR UN APPAREIL POUR LE doSAGE DE LA MATIËRE GRASSE DANS LA CASÉINE PRÉsURE
}

\author{
par M. Mard FOUASSIER \\ Expert-Chimiste près les Tribunaux
}

Dans le numéro 141 (Janvier 1935) de la Revue Le Lait, j'ai lu avec intérêt la description d'un appareil, et celle d'une technique s'y adaptant, pour le dosage de la matière grasse dans la caséine présure, présentées par M. J. Delorme.

L'intérêt que j'ai pris à la lecture de cet article était d'autant plus vif que j'avais antérieurement publié dans cette même Revue (n० 83, mars 1929, p. 269) un article analogue, dans lequel je présentais également un appareil pour l'application d'un même principe d'analyse.

Ce principe et ses modalités ne sont pas une nouveauté : je n'envisagerai donc pas l'attaque chlorhydrique de la substance sur laquelle il repose, mais seulement l'appareil proposé par M. DELoRME, appareil qui se différencie du tube très simple que j'avais indiqué, par l'apport d'un robinet qui supprime le pipettage et permet une décantation de la couche de solvant éthéré.

Je m'abstiendrai de toute critique concernant le dit appareil, qui peut avoir ses avantages dans certains cas industriels, malgré l'imprécision des pesées, mais je rappellerai que dans la méthode que j'ai décrite, - et qui est journellement employée, - j'utilise des tubes gradués, spécialement construits sur mes indications, dans lesquels on effectue l'attaque de la caséine au bain-marie en atmosphère acide, c'est-à-dire que toute diffusion de vapeurs chlorhydriques est évitée par l'adjonction d'un tube de dégagement muni d'une boule et dont la pointe effilée plonge dans de l'eau ; la légère surpression ainsi provoquée n'est pas sans activer l'attaque. Je n'ai pas eu à constater des variations dans les résultats en mainte- 